\title{
COVID-19 Cases Detection: Suspect Case Strategy
}

\author{
Zhongliang Ji ${ }^{1}$, Kailin $\mathrm{Lu}^{2}$, Xuedong Sun ${ }^{1^{*}}$ \\ ${ }^{1}$ Shenzhen University General Hospital, Shenzhen 518000, China. E-mail: 437994009@qq.com \\ ${ }^{2}$ The University of Hong Kong-Shenzhen Hospital, Shenzhen 518000, China
}

Abstract: Background and objective: as COVID-19 has triggered enormous human casualties and disastrous economic loss, the strategies to contain its spread are urgently needed. We aim to assess the value of our suspect case strategy on COVID-19 cases detection and ensure it to be a complement to the RT-PCR test. Materials and methods: from January 23 to April 30, 2020, patients admitted to our emergence isolation ward (EIW) were analyzed in this study. With RT-PCR as a reference standard, all participants were assigned to COVID-19 positive and negative groups. We compared the performance of the suspect case strategy, WBC, LYM, and chest radiography on COVID-19 detection. Results: one hundred twenty-five cases were analyzed in the study period. The receiver operating characteristic (ROC) curve revealed the sensitivity of WBC and LYM on COVID-19 detection was $92.11 \%$ and $76.31 \%$, respectively. The sensitivity of the suspect case was $94.73 \%$, and the sensitivity of chest CT was $53.33 \%$. Statistically significant differences were observed for the suspect case, WBCs, LYM, and chest CT on COVID-19 detection. Discussion: suspect case strategy can help identify false negative RT-PCR tests and be a useful complement to RT-PCR in some countries with a tremendous shortage of RT-PCR agents and specialized laboratory during the COVID-19 outbreak.

Keywords: Suspect Case; COVID-19; RT-PCR; Blood Test; Chest CT

\section{Introduction}

In late December 2019, a novel pathogen, SARS $\mathrm{CoV}-2$, first emerged in China and has evolved into a global pandemic ${ }^{[1-2]}$. COVID-19 is causing a worldwide medical healthy crisis and challenging the global healthcare system $^{[3]}$. Although strict regulations and restrictions have been implemented to contain the virus's spread, confirmed cases and death tolls caused by COVID-19 continue to rise throughout the world ${ }^{[4]}$. These interventions undertook by some countries have a real effect on interrupting transmission and decreasing the confirmed cases; however, strategies against a potential rebound wave of transmission because of the gradual resumption of economic activities are still needed ${ }^{[5-6]}$. COVID-19 is found to have higher transmissibility ${ }^{[7]}$, and the majority of infected patients are asymptomatic or with mild symptoms ${ }^{[8-9]}$. Thus, early detection of positive cases is crucial. RT-PCR serves as the reference standard for COVID-19 diagnosis. However, RT-PCR has suffered from many flaws, such as the long turnaround times, the need for certified laboratories, expensive equipment, trained personnel, and high false-negative results ${ }^{[10-11]}$. The positive rate of RT-PCR for throat swab samples was reported to be about $30 \%$ to $60 \%$ at the initial presentation $^{[12]}$. These flaws constrain RT-PCR as large-scale screening aiming at a rapid and reliable diagnosis of

Copyright (C) 2020 Zhongliang Ji et al.

doi: $10.18686 /$ aem.v9i3.175

This is an open-access article distributed under the terms of the Creative Commons Attribution Non-Commercial License

(http://creativecommons.org/licenses/by-nc/4.0/), which permits unrestricted non-commercial use, distribution, and reproduction in any medium, provided the original work is properly cited. 
patients. An alternative strategy is needed to ensure that potential cases of COVID-19 are identified early and safely.

Recent studies showed that some specific laboratory parameters were changed on COVID-19 patients ${ }^{[13-15]}$. These parameters might be quickly affected by a large variability, and those changes were also observed in healthy individuals. Characteristic radiographic findings have been reported on chest CTs of patients with COVID-19 infection. Chest CT is more sensitive than RT-PCR in the earliest (likely asymptomatic) phases of infection. However, normal chest CTs were also noted in patients with positive RT-PCR results but clinical symptoms. Those limitations also constrain laboratory parameters and chest CT as better COVID-19 detection tools. It is essential for a suspect case strategy helping to optimize the existing medical resources ${ }^{[17]}$. However, its value on COVID-19 detection was uncertain, so we aimed to assess the performance of our suspect case strategy on COVID-19 detection.

\section{Materials and methods}

\subsection{General setting}

Our hospital is located in the south of China and is far away from the COVID-19 outbreak zone. All patients registered in the emergence department with COVID-19 exposure history or fever $\left(\mathrm{T}>37.2^{\circ} \mathrm{C}\right)$ were managed in segregated areas of the ED ("fever clinic"), and complete blood count (CBC) and chest radiographs with COVID-19 nucleic acid test were performed immediately at fever clinic. Subsequently, patients with COVID-19 exposure history or undifferentiated fever $\left(\mathrm{T}>37.2^{\circ} \mathrm{C}\right)$ were admitted into our emergence isolation ward (EIW) for investigations. COVID-19 positive cases detected in IW were transferred to a hospital designated for confirmed cases. Multiple COVID-19 samples would be performed until a positive sample was noted or reached the criteria for discharge when radiographic featured presenting with viral pneumonia. All images would be reviewed by two radiologists independently, and final decisions were reached by consensus. The minimum criteria for discharge were two negative COVID-19 samples taken 24 hours apart with negative symptoms and chest radiographic.

\subsection{Suspect case criteria for COVID-19}

Exposure risk with any two items of clinical features; all three items of clinical features.

(1) Clinical features: (a) fever or respiratory symptoms; (b) imaging features of COVID-19 pneumonia; (c) WBCs $<9.93 \times 10^{9}$ cells $/ \mathrm{L}$ and $\mathrm{LYM}<3.61 \times 10^{9}$ cells $/ \mathrm{L}$.

(2) COVID-19 exposure risk (in the last 14 days before symptom onset): (a) a history of stay at the continuous transmission of local cases area; (b) contacted fever or respiratory symptoms patients belong to the continuous transmission of local cases; (c) contacted with COVID-19 patients; (d) clustered onsets ${ }^{[18]}$.

\subsection{Study design and participants}

With RT-PCR as a reference standard, all participants were assigned to COVID-19 positive and negative groups. We analyzed initial plasma levels of WBCs, NEUT, LYM MON for COVID-19 prediction in each group. WBCs and LYM are potential diagnostic tests based on their AUC results. The performance of suspect case strategy, WBC, LYM, and chest radiography on detecting COVID-19 were assessed. We also compared these selected tests as a potential diagnostic test on COVID-19 detection.

\subsection{Procedures protocol}

Blood samples and COVID-19 nasal swab samples were collected upon patients' arrival, and experienced nurses performed the procedures wearing PPE and N95. All the samples were analyzed in our hospital's laboratory, and the turnover period was 30 minutes for CBC and 6 hours for the COVID-19 nucleic test. Chest CT was performed in a cabin $\mathrm{CT}$ machine immediately after blood and nasal swab samples were collected.

\subsection{Data collection}

The medical records of patients were analyzed by the research team of our department, and epidemiological history, blood results, and radiological data were obtained in data collection forms from electronic medical records. A trained team of physicians reviewed the data. Information records included demographic data, medical history, exposure history, underlying comorbidities, symptoms, laboratory findings, and chest CT results.

\section{Results}

\subsection{Basic characteristics and clinical labora-}




\section{tory findings}

A total of 125 cases were analyzed in the study period and 38 cases $(38 / 125,30 \%)$ were COVID-19 positive whereas $87(87 / 125,70 \%)$ were negative. They were 59 males and 66 females. Their median age was 38 (22-53) years. 108 (86\%) of the patients had COVID-19 exposure risk. $19(15.2 \%)$ of the patients had underlying diseases, such as diabetes (2\%), hypertension (8\%), chronic obstructive pulmonary disease $(2 \%)$, and cardiovascular disease (2\%). Clinical manifestations included fever $(77 / 125,62 \%)$, cough $(67 / 125,54 \%)$, sputum production $(44 / 125,35 \%)$, short of breath $(4 / 125,3 \%)$, myalgia or fatigue $(12 / 125,10 \%)$, headache $(8 / 125,6 \%)$, and diarrhea $(12 / 125,10 \%)$ (Table 1).

\begin{tabular}{|c|c|c|c|c|}
\hline & $\begin{array}{l}\text { All patients } \\
(\mathrm{N}=125)\end{array}$ & $\begin{array}{l}\text { COVID-19 positive } \\
(\mathrm{N}=38)\end{array}$ & $\begin{array}{l}\text { COVID-19 negative } \\
(\mathrm{N}=87)\end{array}$ & P-value \\
\hline \multicolumn{5}{|l|}{ Characteristics } \\
\hline Age, years & $38.0(22.0-53.0)$ & $42.0(25.0-58.0)$ & $36.0(21.0-51.0)$ & 0.57 \\
\hline Sex & & & & 0.115 \\
\hline Men & $59(47 \%)$ & $37(43 \%)$ & $22(58 \%)$ & \\
\hline Women & $66(53 \%)$ & $50(57 \%)$ & $16(42 \%)$ & \\
\hline Exposure risk & $108(86 \%)$ & $34(89 \%)$ & $74(85 \%)$ & 0.512 \\
\hline \multicolumn{5}{|l|}{ Comorbidity } \\
\hline Diabetes & $3(2 \%)$ & $1(3 \%)$ & $2(2 \%)$ & 0.912 \\
\hline Hypertension & $10(8 \%)$ & $4(11 \%)$ & $6(7 \%)$ & 0.495 \\
\hline $\begin{array}{l}\text { Chronic obstructive } \\
\text { pulmonary disease }\end{array}$ & $3(2 \%)$ & $2(5 \%)$ & $1(1 \%)$ & 0.17 \\
\hline Cardiovascular disease & $3(2 \%)$ & $1(3 \%)$ & $2(2 \%)$ & 0.912 \\
\hline \multicolumn{5}{|l|}{ Symptoms } \\
\hline Fever & $77(62 \%)$ & $24(63 \%)$ & $53(61 \%)$ & 0.815 \\
\hline Cough & $67(54 \%)$ & $17(45 \%)$ & $50(57 \%)$ & 0.192 \\
\hline Sputum production & $44(35 \%)$ & $9(24 \%)$ & $35(40 \%)$ & 0.076 \\
\hline Short of breath & $4(3 \%)$ & $2(5 \%)$ & $2(2 \%)$ & 0.390 \\
\hline Myalgia or fatigue & $12(10 \%)$ & $4(11 \%)$ & $8(9 \%)$ & 0.818 \\
\hline Headache & $8(6 \%)$ & $5(13 \%)$ & $3(3 \%)$ & $0.042 *$ \\
\hline Diarrhea & $12(10 \%)$ & $5(13 \%)$ & $7(8 \%)$ & 0.376 \\
\hline Vomit & $2(1 \%)$ & $0(0 \%)$ & $2(2 \%)$ & 0.350 \\
\hline
\end{tabular}

Table 1. Baseline characteristics and symptoms of the study patient

$* P<0.05$ was considered as statistically significant

\subsection{The performance of initial blood param- eters and chest radiography on COVID-19 patients}

Statistically significant differences were observed for WBC, NEUT, and LYM between COVID-19 positive and negative groups (Table 2). The receiver operating characteristic (ROC) curve revealed that the area under the curve (AUC) of WBC, NEUT, LYM, MON were 0.719, 0.630, 0.696, 0.652, respectively (Table 3). WBCs and LYM were chosen as potential diagnostic tests in this study based on their higher AUC results. Youden's index revealed cut-off values (WBC: 8.1150, LYM: 1.6750)
(Figure. 1). Those cut-off values yielded a sensitivity of 92.11\% (95\% confidence interval, $\mathrm{CI}=77.5 \%-97.9 \%$ ) and a specificity of $42.52 \%$ (95\% confidence interval, CI $=32.1 \%-53.6 \%)$ for $\mathrm{WBC}$, and sensitivity of $76.31 \%$ (95\% confidence interval, $\mathrm{CI}=59.4 \%-88.0 \%$ ) and a specificity of $59.77 \%$ (95\% confidence interval, $\mathrm{CI}=$ 48.7\%-70.0\%) for LYM. The sensitivity of chest radiographic scan was $53.33 \%$ (95\% confidence interval, $\mathrm{CI}=$ $38.0 \%-68.0 \%$ ), and the specificity was $82.5 \%$ (95\% confidence interval, $\mathrm{CI}=72.0 \%-89.8 \%$ ) (Table 4).

\subsection{The performance of suspect case strategy on COVID-19 patients}


Sixty $(60 / 125,48 \%)$ patients fulfilled suspect case criteria. $36(36 / 60,60 \%)$ were COVID-19 positive whereas $24(24 / 60,40 \%)$ were negative. The sensitivity of suspect case was $94.73 \%$ (95\% confidence interval, CI $=80.9 \%-99.1 \%)$, and the specificity was $72.41 \%(95 \%$ confidence interval, $\mathrm{CI}=61.6 \%-81.2 \%$ ) (Table 4). Statistically significant differences were observed for suspect cases, WBCs, LYM, and chest radiography on COVID-19 detection (Table 5).

\begin{tabular}{|l|l|l|l|l|}
\hline Parameters & Unit & COVID-19 positive & COVID-19 negative & P value \\
\hline WBC & $\times 109$ cells/L & $5.67 \pm 1.70$ & $7.74 \pm 3.09$ & 0.000 \\
\hline Neutrophils & $\times 109$ cells/L & $3.73 \pm 1.46$ & $4.98 \pm 2.88$ & 0.013 \\
\hline Lymphocytes & $\times 109$ cells/L & $1.45 \pm 1.46$ & $2.04 \pm 1.10$ & 0.003 \\
\hline Monocytes & $\times 109$ cells/L & $0.43 \pm 0.18$ & $0.76 \pm 1.71$ & 0.230 \\
\hline
\end{tabular}

Table 2. Averaged blood parameters of patients

$* P<0.05$ was considered as statistically significant

\begin{tabular}{|l|l|l|l|l|l|l|}
\hline Variable & AUC & SE & P-value & $\mathbf{9 5 \%}$ CI & Youden Index & Cut-off values \\
\hline WBC & 0.719 & 0.047 & $0.000^{*}$ & 0.628 to 0.811 & 0.358 & 7.7000 \\
\hline LYM & 0.696 & 0.050 & $0.001^{*}$ & 0.598 to 0.793 & 0.346 & 1.6500 \\
\hline NEUT & 0.630 & 0.051 & $0.021^{*}$ & 0.530 to 0.731 & 0.239 & 4.3000 \\
\hline MON & 0.652 & 0.53 & $0.007^{*}$ & 0.547 to 0.765 & 0.236 & 0.4950 \\
\hline
\end{tabular}

Table 3. Discriminative performance of prediction for COVID-19

$* P<0.05$ was considered as statistically significant

\begin{tabular}{|l|l|l|l|l|l|l|l|l|}
\hline \multirow{2}{*}{ Test } & \multicolumn{3}{|l}{ Results(n) } & \multicolumn{2}{l|}{ Test performance (\%) } \\
\cline { 2 - 10 } & TP & FP & TN & FN & $\begin{array}{l}\text { Sensitivity } \\
(\mathbf{9 5 \%} \text { CI) }\end{array}$ & $\begin{array}{l}\text { Specificity } \\
(\mathbf{9 5 \%} \text { CI) }\end{array}$ & PPV (95\% CI) & NPV (95\% CI) \\
\hline Suspect case & 36 & 63 & 24 & 2 & $94.73(80.9-99.1)$ & $72.41(61.6-81.2)$ & $60.00(46.5-72.2)$ & $96.92(88.4-99.5)$ \\
\hline WBC & 35 & 50 & 37 & 3 & $92.11(77.5-97.9)$ & $42.52(32.1-53.6)$ & $41.18(30.8-52.4)$ & $92.50(78.5-98.0)$ \\
\hline LYM & 29 & 35 & 52 & 9 & $76.31(59.4-88.0)$ & $59.77(48.7-70.0)$ & $45.31(33.0-58.2)$ & $85.24(73.3-92.6)$ \\
\hline CT & 24 & 14 & 66 & 21 & $53.33(38.0-68.0)$ & $82.5(72.0-89.8)$ & $63.16(46.0-77.7)$ & $75.86(65.3-84.1)$ \\
\hline
\end{tabular}

Table 4. Prediction performance of COVID-19 with different screening test

$C T=$ chest $C T \quad T P=$ true positive, $T N=$ true negative, $F P=$ false positive, $F N=$ false negative, $P P V=$ positive predictive value, $N P V=$ negative predictive value

\begin{tabular}{|l|l|l|l|l|l|l|}
\hline $\begin{array}{l}\text { All patients } \\
\text { N=125 }\end{array}$ & $\begin{array}{l}\text { Suspect case } \\
\mathbf{N = 6 0}\end{array}$ & $\begin{array}{l}\text { WBC }(<\mathbf{7 . 7 0 0 0}) \\
\mathbf{N = 8 5}\end{array}$ & $\begin{array}{l}\text { LYM(<1.6500) } \\
\mathbf{N = 6 4}\end{array}$ & $\begin{array}{l}\text { Positive CT } \\
\mathbf{N = 3 8}\end{array}$ & $\begin{array}{l}\chi^{\mathbf{2}} \\
\text { statistic }\end{array}$ & Pvalue \\
\hline $\begin{array}{l}\text { COVID-19 } \\
\text { positive }\end{array}$ & 36 & 35 & 29 & 24 & 8.237 & $0.041^{*}$ \\
\cline { 1 - 1 } $\begin{array}{l}\text { COVID-19 } \\
\text { negative }\end{array}$ & 24 & 50 & 35 & 14 & \\
\hline
\end{tabular}

Table 5. Selected screening test on COVID-19 detection analysis

$C T=$ chest $C T, * P<0.05$ was considered as statistical significant

\section{Discussion}

Early diagnosis and isolate patients with COVID-19 exposure history or respiratory symptoms are crucial to containing the pandemic. The COVID-19 exposure risk and clinical features are associated with a positive
SARS-CoV-2 test ${ }^{[19]}$. In our study, 34 (89\%) COVID-19 patients had positive exposure history. However, there was no difference in exposure history between the two groups. The most common symptoms at the onset of illness were fever (24 [63\%]), cough (17 [45\%]), sputum production (9 [24\%]). Less common symptoms were 
headache, diarrhea, and vomiting (Table 1). However, there was also no difference in significant clinical findings between the two groups except the headache. The reason for the headache discrepancy is likely to be the low number of patients enrolled in our study. So it is difficult to differentiate and screen patients just with exposure history and clinical symptoms in the emergency department setting.

Certain blood biochemical indexes were associated with COVID-19 patients $^{[20-21]}$. The most common laboratory abnormalities observed in this study were depressed total WBCs, LYM, NEUT (Table 2). They are consistent with the current study ${ }^{[22]}$, and WBCs and LYM achieved excellent diagnosis performance with AUC = 0.719 and 0.630 (Table 3). With RT-PCR results as a reference, our suspect case had a better sensitivity and specificity on COVID-19 detection compared with WBCs and LYM. Better performance also was found in positive predictive value and negative predictive value, respectively. These biochemical indexes need to incorporate other diagnostic factors to achieve excellent performance on COVID-19 detection.

Chest CT was reported to be a valuable diagnostic tool for COVID-19 ${ }^{[23]}$. Characteristic CT features of COVID-19 patients were described in the recently published literature ${ }^{[24-26]}$. In our study, about $63 \%$ (24/38) cases had typical initial CT features consistent with COVID-19 positive RT-PCR. The sensitivity and specificity of chest CT scan on COVID-19 detection were $53.33 \%(95 \% \mathrm{CI}=38.0 \%-68.0 \%)$ and $82.5 \%$ (95\% CI $=72.0 \%-89.8 \%$ ). One study's results described by Ai T, etc.al, were better than ours ${ }^{[27]}$. The discrepancy between the two results is likely to be different from enrolled patients and settings. Ai's study was observed in Wuhan with a higher COVID-19 prevalence than our city cause; this study enrolled in patients who came from the emergence department. CT can decrease the chance of false-negative results in the RT-PCR assay. Many potential COVID-19 patients with first or second negative RT-PCR tests were found and reported with positive CT findings in our clinical practice ${ }^{[28-31]}$. Therefore, multiple swab samples were collected for COVID-19 detection until positive samples were noted or met the criteria for discharge. Positive chest CT had a good performance on COVID-19 detection.

Given one COVID-19 case's inappropriate man- agement in the emergence department can result in large numbers of individuals exposure and potential infection $^{[32]}$. Isolating all patients with respiratory symptoms or screening all patients by one diagnostic tool may not detect all COVID-19 patients. The suspected case strategy incorporated epidemiology risk and clinical features combining with WBCS, LYM, and chest CT, it can compensate for the above shortcoming, and its value on COVD-19 detection is better than any single diagnostic tool (Table 5). The suspect case also can be a potential diagnostic tool for COVID-19.

\section{Limitation}

Because enrolled cases were limited in the isolation room, we cannot extend the detection value of the suspect case strategy to all the potential COVID-19 patients in the emergence room. Future studies should include all potential cases in the ER, and multicenter studies will optimize the practical applicability of the suspect case strategy.

\section{Conclusion}

A suspect case strategy can help to ensure appropriate triage and optimize testing resources, but it also can be a potential diagnostic tool for COVID-19 detection. Combining appropriate specific hematological parameters with chest radiography can be helpful to identify false positive/negative RT-PCR tests. Blood test analysis and chest radiography might be used as an alternative to RT-PCR for identifying COVID-19 positive patients in those countries which suffer from a massive shortage of RT-PCR reagents and specialized laboratory.

\section{Funding statement}

This research was funded by Sanming Project of Medicine in Shenzhen (No: SZSM201911007). The data used to support the findings of this study are available from the corresponding author upon request.

\section{References}

1. Zhou P, Yang X, Wang X, et al. A pneumonia outbreak associated with a new coronavirus of probable bat origin. Nature 2020; 579(7798): 270-273.

2. Pongpirul WA, Pongpirul K, Ratnarathon AC, et al. Journey of a Thai taxi driver and novel coronavirus. N Engl J Med. 2020; 382(11): 1067-1068. 
3. WHO. Rolling updates on coronavirus disease (COVID-19): WHO characterizes COVID-19 as a pandemic [Internet]. Available from: https://www.who.int/emergencies/diseases/novelcor onavirus-2019/events-as-they-happen 221-228.

4. Chinazzi M, Davis JT, Ajelli M, et al. The effect of travel restrictions on the spread of the 2019 novel coronavirus (COVID-19) outbreak. Science 2020; 136-148.

5. Leung K, Wu J, Liu D, et al. First-wave COVID-19 transmissibility and severity in China outside Hubei after control measures, and second-wave scenario planning: A modelling impact assessment. The Lancet 2020; 395(10233): 1382-1393.

6. Kraemer M, Yang CH, Gutierrez B, et al. The effect of human mobility and control measures on the COVID-19 epidemic in China. Science 2020; 1116.

7. Huang C, Wang Y, Li X, et al. Clinical features of patients infected with 2019 novel coronavirus in Wuhan, China. The Lancet 2020; 395(10223): 497506.

8. Wang D, Hu B, Hu C, et al. Clinical characteristics of 138 hospitalized patients with 2019 novel coronavirus-infected pneumonia in Wuhan, China. The Journal of the American Medical Association 2020; 56-63.

9. Prem K, Liu Y, Russell TW, et al. The effect of control strategies to reduce social mixing on outcomes of the COVID-19 epidemic in Wuhan, China: A modelling study. Lancet Public Health 2020; 8795.

10. Li Z, Yi Y, Luo X, et al. Development and clinical application of a rapid IgM-IgG combined antibody test for SARSCoV-2 infection diagnosis. Journal of Medical Virology 2020; 56-58.

11. Lippi G, Simundic AM, Plebani M. Potential preanalytical and analytical vulnerabilities in the laboratory diagnosis of coronavirus disease 2019 (COVID-19). Clinical Chemistry and Laboratory Medicine 2020; 102-110.

12. Yang Y, Yang M, Shen C, et al. Evaluating the accuracy of different respiratory specimens in the laboratory diagnosis and monitoring the viral shedding of 2019-nCoV infections. 2020. 88-95.

13. Fan BE, Chong VC, Chan SS, et al. Hematologic parameters in patients with COVID-19 infection. American Journal of Hematology 2020; 98-103.

14. Lippi G, Plebani M. Laboratory abnormalities in patients with COVID-2019 infection. Clinical Chemistry and Laboratory Medicine 2020.

15. Ai T, Yang Z, Hou H, et al. Correlation of chest CT and RT-PCR testing in coronavirus disease 2019 (COVID-19) in China: A report of 1014 cases. Radiology 2020; 108-112.
16. Wee LE, Fua TP, Chua YY, et al. Containing COVID-19 in the emergency room: The role of improved case detection and segregation of suspect cases. Academic Emergency Medicine 2020; 27(5): 379-387.

17. Office of State Administration of Traditional Chinese Medicine. Notice on the issuance of a programme for diagnosis and treatment of novel coronavirus (2019-nCoV) infected pneumonia [Internet]. Available from: http://bgs.satcm.gov.cn/.

18. Sun Y, Koh V, Marimuthu K, et al. Epidemiological and clinical predictors of COVID-19. Clinical Infectious Diseases 2020; 71(15).

19. Liu Y, Yang Y, Zhang C, et al. Clinical and biochemical indexes from 2019-nCoV infected patients linked to viral loads and lung injury. Nature Public Health Emergency Collection 2020; 63(3): 364 374.

20. Ferrari D, Motta A, Strollo M, et al. Routine blood tests as a potential diagnostic tool for COVID-19. Clinical Chemistry and Laboratory Medicine 2020; 68-73.

21. Dai WC, Zhang HW, Yu J, et al. CT imaging and differential diagnosis of COVID-19. Canadian Association of Radiologists Journal 2020.

22. Lei J, Li J, Li X, et al. CT imaging of the 2019 novel coronavirus (2019-nCoV) pneumonia. Radiology 2020; 295(1): 200257.

23. Shi H, Han X, Zheng C. Evolution of CT manifestations in a patient recovered from 2019 novel coronavirus (2019-nCoV) pneumonia in Wuhan, China. Radiology 2020; 295(1): 200269.

24. Pan Y, Guan H, Zhou S, et al. Initial CT findings and temporal changes in patients with the novel coronavirus pneumonia (2019-nCoV): A study of 63 patients in Wuhan, China. Eur Radiol. 2020.

25. Zhifeng J, Feng A, Li T. Consistency analysis of COVID-19 nucleic acid tests and the changes of lung CT. J Clin Virol. 2020; 127: 104359.

26. Huang P, Liu T, Huang L, et al. Use of chest CT in combination with negative RT-PCR assay for the 2019 novel coronavirus but high clinical suspicion. Radiology 2020; 295(1): 22e3.

27. Ruan Z, Gong P, Han W, et al. A case of 2019 novel coronavirus infected pneumonia with twice negative 2019-nCoV nucleic acid testing within 8 days. Chinese Medical Journal 2020; 133(12): 14871488 .

28. Zhou Y, Yang L, Han M, et al. Case report on early diagnosis of COVID-19. Disaster Medicine and Public Health Preparedness 2020; 1-4.

29. Ng KQ, Poon BH, Puar THK, et al. COVID-19 and the risk to healthcare workers: A case report. Annals of Internal Medicine 2020. 